\title{
La asociatividad como factor de desarrollo en la agenda pública del departamento del Quindío: El caso del sector agropecuario y agroindustrial*
}

\author{
Associativity as a development factor in the public agenda \\ of the department of Quindío: The case of the agricultural \\ and agroindustrial sector
}

\section{Associatividade como um desenvolvimento na agenda pública de Quindio: $\mathbf{0}$ caso do setor agrícola e agroindustrial}

\author{
Lina Marcela Sánchez-Vásquez \\ Estudiante de Doctorado en Administración de Negocios, Atlantic International University. Magíster en Desarrollo Sostenible y Medio \\ Ambiente, Universidad de Manizales. Docente Investigadora Adscrita al Grupo de investigación Grupo de investigación en Desarrollo y \\ Crecimiento Económico Regional GIDECER, Escuela de Administración de Corporación Unificada Nacional CUN \\ mariana3032@gmail.com
}

\begin{abstract}
Resumen
El desarrollo concebido como un estado de plena realización de la vida de un territorio, requiere la confluencia de factores que permitan el logro de este propósito, para ello los planes de desarrollo se convierten en una herramienta para la priorización de necesidades en función de los territorios y sus posibilidades de recursos. El departamento del Quindío no es ajeno a esta realidad y ha establecido la priorización de apuestas productivas a través de sus instrumentos de política pública, siendo algunos de estos la Agenda Interna de Productividad y Competitividad, el Plan de Desarrollo Departamental del Quindío 20122015 (años de referencia para el análisis) y Agenda de Ciencia, Tecnología e Innovación, entre otros. Considerando al departamento como parte del denominado eje cafetero y con el sector agroindustrial como dinamizador de este planteamiento, subyace la inquietud con referencia a las posibilidades de fortalecimiento y desarrollo del sector al considerar la cultura asociativa como un factor clave en el cumplimiento de los planes, programas y proyectos descritos en la agenda de desarrollo local. Surge así la inquietud por la contribución que realizó a este propósito el Plan de Desarrollo del Departamento del Quindío 2012-2015 a la cultura asociativa del sector agropecuario y agroindustrial.
\end{abstract}

* Cómo citar: Sánchez-Vásquez, L. M. (2016). La asociatividad como factor de desarrollo en la agenda pública del departamento del Quindío: El caso del sector agropecuario y agroindustrial. RevistaLibre Empresa, 13(2), 57-71 http://dx.doi.org/10.18041/libemp.2016.v13n2.26204 


\section{Palabras clave}

Desarrollo, asociatividad, política pública, sector agroindustrial.

\section{Abstract}

The development conceived as a state of full realization of the life of a territory requires the confluence of factors that allow achieving this purpose, for this, development plans become a tool for the prioritization of needs in terms of territories and possibilities of resources. Quindío is no stranger to this reality and has established prioritizing productive bets through their public policy instruments, some of these Internal Agenda for Productivity and Competitiveness, the Departmental Development Plan 2012-2015 Quindío ( reference years for analysis) and agenda Science, Technology and Innovation, among others. Considering the department as part of so-called coffee axis and the agro industrial sector as a catalyst for this approach underlies the concern with reference to the possibilities of strengthening and development of considering the associative culture as a key factor in the implementation of plans, programs sector projects described in the local development agenda, the concern arises for the contribution he made to this purpose the development plan 2012-2015 Quindío the associative culture of the agricultural and agro industrial sector.

\section{Keywords}

Development, associativity, public policy, agroindustrial sector.

\section{Resumo}

O desenvolvimento concebido como um estado de plena realização da vida de um território exige a confluência de fatores que permitem atingir este objectivo, por isso, os planos de desenvolvimento se tornar uma ferramenta para a priorização das necessidades em termos de territórios e possibilidades de recursos. Quindio não é estranho a esta realidade e estabeleceu priorizando apostas produtivas através de seus instrumentos de políticas públicas, alguns destes Agenda Interna de Produtividade e Competitividade, o Desenvolvimento Departamental Plano 2012-2015 Quindio (anos de referência para a análise) e agenda de ciência, tecnologia e inovação, entre outros. Considerando o departamento como parte do chamado eixo do café e do setor agroindustrial como um catalisador para esta abordagem está subjacente à preocupação com referência às possibilidades de fortalecimento e desenvolvimento de considerar a cultura associativa como um factor-chave para a implementação de planos, programas sectoriais projectos descritos na agenda de desenvolvimento local, a preocupação surge para a contribuição que ele fez para o efeito, o plano de desenvolvimento 2012-2015 Quindio a cultura associativa do sector agrícola e agro-industrial.

\section{Palavras chave}

Desenvolvimento, associatividade, política pública, o setor agroindustrial. 


\section{Introducción}

Este documento aborda un acercamiento preliminar a la forma como contribuye la política pública departamental y sus priorizaciones en la inclusión de este ítem, dentro de la Agenda de Desarrollo del Departamento.

La diversidad económica y geográfica en Colombia es parte fundamental de la generación de desarrollo en los diferentes sectores económicos. En el caso del sector ganadero, su fortalecimiento y participación dentro del PIB nacional se sustenta en la presencia concentrada de esta actividad económica en cinco departamentos (Antioquia, Córdoba, Casanare, Meta y Santander) según el informe de EMIS (2015). Su desempeño está mediado por factores tales como tipo de mercados objetivo, uso de tecnología en su desarrollo, posibilidad de inclusión de principios de economía de escala que tienen influencia significativa en las estructuras de costos, los desarrollos normativos vigentes y próximos, la regulación de precios, entre otros que condicionan y advierten factores a considerar en el proceso productivo en consonancia con las realidades del contexto del sector.

La influencia del sector ganadero en la economía nacional, le ubica en la posición 33 dentro del total de sectores nacionales: 85, lo cual implica una influencia significativa dentro de los renglones que soportan la estructura económica colombiana. Aunada a lo anterior la confluencia de actividades de significativa importancia como la agricultura, la caza, la silvicultura y la ganadería propiamente en este sector, proporcionan elementos base para la necesidad de construcción económica de políticas orientadas a su desarrollo y consolidación, no solo en la escena nacional sino también en el contexto del comercio exterior, en consonancia con la política de internacionalización económica y su dinamización a través de los tratados de libre comercio.

Al ser incluidos como se visualiza en la Agenda de Desarrollo Nacional y Departamental, es necesario conocer la manera en que se permite su fortalecimiento mediante la participación y vinculación del capital social de los territorios, en este caso en el departamento del Quindío.

\section{Desarrollo}

\section{El sector agroindustrial en la Agenda de Desarrollo Económico Departamental, periodo 2012-2015}

El Plan de Desarrollo Departamental se aprobó con la Ordenanza Número 018 del 30 de mayo de 2012, "Por medio del cual se adopta el Plan de Desarrollo Departamental 2012-2015 "Gobierno Firme por un Quindío Más Humano”; este documento resalta en el diagnóstico inicial al departamento como parte de la región conocida como Eje Cafetero, conformada por tres departamentos: Caldas, Risaralda y Quindío. Se destaca que la producción agrícola del departamento se concentra en cultivos de tipo permanente y dentro de ellos, solo tres cultivos específicos (plátano, cítricos y café) registran más del 80\% del total de producción, según cifras del 2010. El plátano para consumo es, sin duda, el producto líder en modalidad independiente o intercalada. Quindío es el primer productor de plátano para consumo en Colombia. (Departamento del Quindío, 2012 ). 
En la cultura agrícola y en general en la cultura cafetera del departamento, constituye un factor fundamental el relacionamiento social, basado en la confianza que permite potenciar y hacer más competitivo este sector económico en la región y en el país. Por tanto, en reconocimiento de estas características socioculturales, se ha visionado el territorio como el producto de una construcción social que permite reconocer sus condiciones, limitaciones, potencialidades y oportunidades de desarrollo.

En este sentido, se reconoce dentro de la Agenda de Desarrollo Departamental 20122015 la integración de cinco dimensiones que estructuran desde la política sociocultural, socioeconómica, ambiental, territorial e institucional. Dentro de los enfoques desarrollados en el Plan de Desarrollo "Gobierno Firme por un Quindío más Humano" se define a las personas como centro del desarrollo, con estrategias de desarrollo diferenciados por ciclo vital, teniendo en cuenta la garantía de derechos, el cumplimiento con los Objetivos de Desarrollo del Milenio, la perspectiva de género y la correspondencia con una visión integral del territorio. En el capítulo tres el Plan de Desarrollo advierte la importancia del sector agroindustrial para cumplir con el propósito de convertir al Quindío en un departamento promisorio, competitivo e innovador.

Para este propósito, y visto porcentualmente por ramas de actividad económica, se puede observar cómo el agro (agricultura, ganadería, caza, silvicultura y pesca) ha disminuido su participación porcentual en el PIB departamental, y el comercio, incluyendo industria hotelera (comercio, reparación, restaurantes y hoteles), ha aumentado la suya.

La generación de valor es baja y pese a ser la agroindustria una de las principales apuestas productivas para el desarrollo del departamento, la evolución del sector no refleja dinamismo. No obstante, la generación de valor es una apuesta dentro de la Agenda de Desarrollo, por cuanto la cultura cafetera, arraigada al territorio se convierte en factor dinamizador del desarrollo y garante de generar un alcance social más amplio de acceso a posibilidades de empleabilidad y aprovechamiento de los recursos disponibles.

De otra parte, el café -producto insignia del departamento- ha venido perdiendo terreno frente a otros cultivos que no necesariamente son intensivos en aplicación de nuevas tecnologías e innovación. Para el 2000, el Producto Interno Bruto derivado de la actividad cultivo de café ascendía a \$165 mil millones y para 2010 el valor cayó a \$80 mil millones en términos constantes de 2005. Por el contrario, el PIB asociado a otros productos agrícolas y el PIB pecuario ha aumentado, pasando de \$163 y \$120 mil millones en 2000 a \$206 y \$176 mil millones en 2010, respectivamente. Estos cambios están en función de las necesidades de apoyo gubernamental, institucional y la renovación de cultivos que reducen la productividad en el sector.

En términos de uso del suelo la producción agrícola del departamento se concentra en cultivos de tipo permanentes y dentro de ellos, solo tres cultivos específicos (plátano, cítricos y café) explican más del 80\% del total de producción, según cifras del 2010. El plátano para consumo es, sin duda, el producto líder en modalidad independiente o intercalada. Quindío es el principal productor de plátano para consumo en Colombia; 
seguido por Arauca, Caldas y Antioquia. El incremento progresivo del área dedicada al cultivo de plátano en el Quindío fue impulsado por la campaña de diversificación para medianos y pequeños caficultores, que en principio, acogieron la siembra de plátano como producto asociado al café.

En general, en los énfasis productivos, tanto agrícolas como pecuarios, de los municipios del departamento no se evidencia un claro perfil productivo que, con base en un ordenamiento estratégico de la producción, aproveche y potencialice las ventajas competitivas y permita construir encadenamientos productivos de alto valor agregado. Urge entonces la revisión de la necesidad de generar una cultura asociativa como factor que posibilite el desarrollo, la competitividad y la productividad del sector agroindustrial en el departamento.

Acorde con lo anterior, en el Plan de Desarrollo se han propuesto componentes estratégicos enfocados al programa de desarrollo rural, con subprogramas tales como: planeación del territorio para el desarrollo rural, competitividad rural, producción agropecuaria sostenible, seguridad alimentaria y, de manera complementaria, el programa de fortalecimiento empresarial y gremial del sector.

\section{La asociatividad y su importancia en el desarrollo}

Diferentes autores e instituciones asociados con el desarrollo han realizado sus aportes teóricos a la manera como el capital social y su base, la confianza, pueden convertirse en motores y potencializadores del desarrollo de los territorios.

La asociatividad, definida por (Sencilio, 2008) Putnam son los aspectos de las organizaciones sociales, tales como las redes, las normas y la confianza que permiten la acción y la cooperación para el beneficio mutuo.

Por su parte, la Organización para la Cooperación y el Desarrollo Económico (OCDE, 2009) lo define como las redes junto con normas, valores y opiniones compartidas que facilitan la cooperación, dentro y entre los grupos. Los aspectos relevantes del concepto son: La confianza entre los miembros funciona mejor cuando existen relaciones horizontales (redes horizontales) sin jerarquía obligatoria (asociaciones, cooperativas y redes informales de vecindad), a diferencia de las relaciones verticales (redes verticales) conformadas por las familias, las empresas y la religión. En las relaciones horizontales hay confianza mutua y cooperación y esta genera asociatividad.

En un estudio realizado por Londoño Franco \& Sánchez Vásquez (2016), la definen como la actitud responsable, propositiva y justa que emerge entre varias personas, con la convicción de que el interés general prima sobre los intereses individuales, a fin de lograr un propósito común en el que tanto la integración como la convivencia, la comunicación y el respeto son pilares fundamentales para la construcción de confianza, progreso y mejoramiento de la calidad de vida de una comunidad o región. Se reconoce en este contexto el valor que para la generación de desarrollo tiene el capital social, entendido como el conjunto de relaciones enmarcadas en aspectos culturales, sociales, económicos, geográficos y políticos que se enfocan en fines comunes para beneficio de la colectividad. 
El BID señala el Capital Social como el conjunto de normas de confianza, valores, actitudes y redes entre personas e instituciones en una sociedad, que define el grado de asociatividad entre los diferentes actores sociales y facilita acciones colectivas y de cooperación. Este concepto se relaciona con confianza, asociatividad, conciencia cívica y valores éticos predominantes que, en conjunto, facilitan o dificultan el desarrollo de determinada sociedad (BID, citado por Valleys 2009).

De lo expresado por los autores e instituciones puede inferirse la influencia que tiene como motor para el desarrollo la generación de asociatividad y la protección del capital social en un territorio. De ahí la necesidad de que este sea un eje que se incorpore en las agendas de desarrollo.

Raúl Atria (2003) expresa que es necesaria una estrategia de asociatividad; es decir, acciones tendientes a expandir o fortalecer la trama o el alcance de las redes en que participan los miembros del grupo, potenciando su cooperación con otros grupos mediante nuevos enlaces de las redes.

Un factor decisivo de la cohesión social es la cultura y su relación directa con la asociatividad: en la cultura las personas pueden reconocerse mutuamente, cultivarse, crecer en conjunto y desarrollar la autoestima colectiva. El capital social y la cultura pueden contribuir al desarrollo si se crean las condiciones adecuadas o, por el contrario, su desconocimiento puede obstaculizar el camino hacia el desarrollo.

Las políticas de desarrollo requieren incluir estrategias para el desarrollo del capital social para ampliarlo, para lo cual según Atria (2003), podría acudirse al empoderamiento esto es, liderazgo y apropiación de algunos miembros del grupo, recurrir a una estrategia de asociatividad, expandiendo las redes de participación de los miembros para la cooperación y colaboración concertada en reconocimiento del ethos, formas de trabajo, valores cívicos y normas a seguir entre comunidades, organizaciones y redes con propósitos comunes.

\section{Metodología}

La investigación asume un enfoque mixto con una fase cualitativa en la recopilación y análisis normativo, posteriormente cuantitativo, para la revisión de la tendencia histórica en el comportamiento de aspectos económico financieros del sector, con una investigación de tipo empírico, analítica y un método deductivo.

Se acude en principio a la recopilación normativa, expedida para el periodo en mención, de manera complementaria al sector agroindustrial, a partir del informe sectorial ganadero para el año 2015 y con referencia a la información histórica comprendida en el mismo entre los años 2010 a 2014 se parte de las cifras extraídas de la base de datos Emis Benchmark, para comparar el desempeño del sector con una empresa específica, siendo ésta la empresa Cerdos del Valle S.A., empresa líder durante el periodo de análisis, para comparar su comportamiento en cuanto a crecimiento en ventas, márgenes sobre ventas, 
generación de utilidad de caja, rentabilidad y liquidez, usando para ello indicadores financieros, graficación y análisis contrastado de resultados, que permitan inferir las relaciones existentes entre las variables del contexto del negocio y los resultados financieros como expresión de las decisiones organizacionales.

\section{Discusión de resultados}

\section{Consideraciones en torno a la cultura asociativa como factor de desarrollo en el sector agroindustrial del departamento del Quindío}

Dentro de las política departamentales se hace evidente el interés e inclusión del trabajo asociativo como priorización del desarrollo, en lo referido al sector agroindustrial, esto se traduce en apoyos gubernamentales por la vía de capacitaciones en emprendimiento y empresarismo, agricultura por contrato, apoyo a proyectos asociativos, fomento a la creación de asociaciones rurales, acompañamiento en convocatorias para la obtención de recursos del gobierno nacional y de cooperación internacional.

Estas estrategias para la garantía de asociatividad son posibles sólo si la cultura e idiosincrasia quindiana permiten el trabajo cooperado y son basados en relaciones de confianza, por tanto, además de convertirse en planes y programas que se traducen proyectos hacia la sociedad, es necesario invertir la forma de producción de la política pública realizándola desde la base, esto es, con procesos de autodiagnóstico que le permitan al gobierno departamental a la vez que conducir los recursos a necesidades ciertas, capitalizar la experiencia colectiva de quienes hacen parte de los sectores promisorios en la agroindustria quindiana.

\section{El sector ganadero en cifras}

En la economía nacional, el sector ha reportado un incremento en su participación dentro del PIB, con un incremento del $0,6 \%$, tendencia que ha sido registrada en los últimos años, el sector ha tenido una dinámica variable, especialmente entre los años 2014 a 2015 derivada de factores externos tales como los efectos del cambio climático, las políticas de producción gremiales y la reducción en el consumo del principal comprador exterior, Venezuela.

En el Gráfico 1 se puede observar la participación del sector en el PIB nacional, alcanzó su máximo en el año 2004 como fuente generadora de ingresos para el país, no obstante por efectos de la variabilidad en la tasa de cambio, el alza en insumos agrícolas y la desaceleración de las exportaciones del sector, entre los años 2009 y 2010 decreció la participación dentro del aporte al PIB nacional. Para los años subsiguientes, la diversificación de los subsectores pecuario y caza, así como especies menores ha permitido que se obtenga una representatividad del 6,12\% en el PIB nacional. 


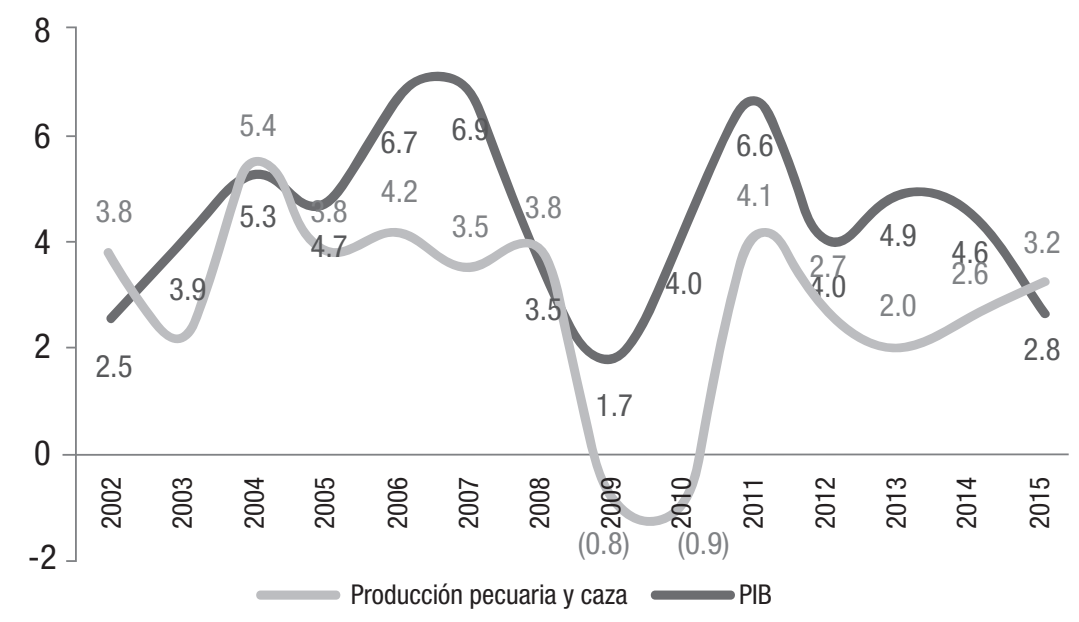

Gráfico 1. Tasa de crecimiento del PIB Producción pecuaria y caza y del PIB

Fuente: DANE. Datos al primer trimestre de 2015.

\section{El liderazgo en el sector: Factores clave de éxito}

Considerando el panorama anterior, las organizaciones toman sus decisiones de producción, financiación, inversión y gestión a partir de las oportunidades y amenazas, así como debilidades y fortalezas que el sector ofrece en su conjunto. La incorporación, en el caso del sector ganadero, de variables externas tales como: la admisibilidad sanitaria para llegar a compradores de países, los precios internacionales y la actual tasa de cambio, la generación de institucionalidad y políticas gremiales y la introducción de tecnología de bajo costo, convierten en puntos a favor las expectativas de desempeño empresariales, haciendo frente a la ocurrencia de posibles amenazas derivadas de la actividad económica, tales como los efectos adversos del cambio climático (sequías y/o intensidad de las lluvias), inseguridad por fenómenos como extorsiones, aumento de la producción en países vecinos, sacrificio y comercialización clandestina.

La lectura del contexto en que operan las organizaciones puede convertirse en una estrategia diferenciadora cuando logra incorporarse en la empresa en su conjunto. Así lo advierte el informe de EMIS (2015), al indicar que la apreciación del mayor o menor liderazgo de las empresas en un mercado particular o sectorial, suministra elementos de juicio valiosos que coadyuvan a entender el grado de competitividad, implicaciones de la aplicación de políticas gubernamentales, estructura de su cadena productiva, comportamiento de los precios, grado de especialización del factor trabajo, barreras de entrada a nuevas empresas, tamaño de sus activos, grado de acceso a las fuentes de financiación, información transmitida al mercado, etc. Todo ello orientado a comprender mejor los riesgos y oportunidades que tienen las empresas en términos de su crecimiento, estabilidad, solidez o insolvencia.

El análisis en este caso se centra en la empresa líder para el año 2014: Cerdos del Valle S.A. (Cervalle) con \$139.196 millones y el 14,7\% del total de las ventas del sector, una empresa cuya operación se caracteriza por la alta tecnificación y especialización en el ramo de producción 
tecnificada y transformación desde la fábrica de alimentos concentrados, pasando por el proceso de cría en las granjas, la sala de procesamiento de carnes frescas, frías y con valor agregado. Dado que su actividad se centra en la diferenciación en el mercado la innovación se convierte en un aspecto fundamental para su posicionamiento y liderazgo en el sector.

Como punto de partida para el entendimiento de la estrategia organizacional, a continuación se presenta el análisis del desempeño financiero de la empresa contrastado con el comportamiento del sector.

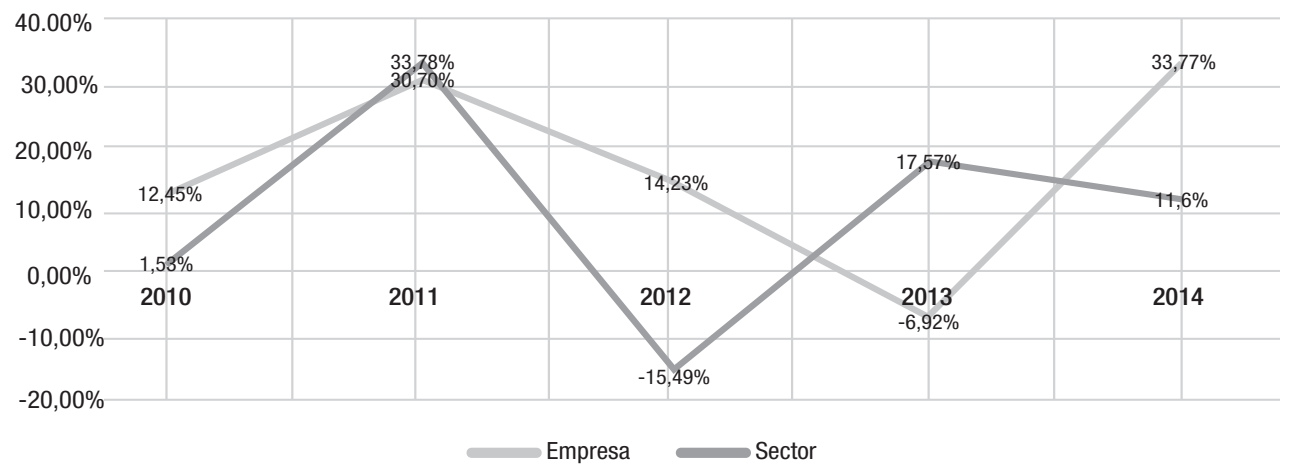

Gráfico 2. Desempeño financiero de Cervalle

Fuente: El autor

El comportamiento en cuanto a crecimiento en ventas del sector entre los años 2010 a 2014 presenta picos y declives, especialmente en lo referido a los años 2011 y 2013 pasando de un incremento del $33 \%$ en el sector y un $30 \%$ en la empresa, a un $17,57 \%$ y un $-6,92 \%$ respectivamente, para el año 2013. Lo anterior como producto de la reducción en la demanda internacional de su principal consumidor: Venezuela. De igual forma se puede evidenciar la tendencia de la organización al alza durante el año 2014, que excede en un $22 \%$ la tendencia del sector.

\section{Tabla 1.}

Indicadores de crecimiento

\begin{tabular}{|c|c|c|c|c|c|c|}
\hline INDICADOR & & 2010 & 2011 & 2012 & 2013 & 2014 \\
\hline \multirow{2}{*}{$\begin{array}{c}\text { Crecimiento / } \\
\text { Disminución en } \\
\text { Activos }\end{array}$} & Empresa & $17,83 \%$ & $11,67 \%$ & $44,19 \%$ & $29,09 \%$ & $25,13 \%$ \\
\hline & Sector & $-29,94 \%$ & $71,13 \%$ & $-25,97 \%$ & $8,58 \%$ & $20,57 \%$ \\
\hline \multirow{2}{*}{$\begin{array}{r}\text { Crecimiento / } \\
\text { Disminución en } \\
\text { Utilidad Neta }\end{array}$} & Empresa & $49,23 \%$ & $172,58 \%$ & $938,59 \%$ & $-98,13 \%$ & $13,30 \%$ \\
\hline & Sector & $-28,43 \%$ & $181,30 \%$ & $21,04 \%$ & $106,10 \%$ & $-12,37 \%$ \\
\hline \multirow{2}{*}{$\begin{array}{l}\text { Crecimiento / } \\
\text { (Disminución) } \\
\text { del Patrimonio }\end{array}$} & Empresa & $15,71 \%$ & $27,45 \%$ & $69,55 \%$ & $-2,49 \%$ & $-10,92 \%$ \\
\hline & Sector & $2,16 \%$ & $91,12 \%$ & $-31,93 \%$ & $7,06 \%$ & $21,51 \%$ \\
\hline
\end{tabular}

Fuente: El autor 
De manera complementada se describe en la Tabla 1 el desempeño en cuanto a crecimiento de activos, mientras el sector ha tenido resultados negativos la rentabilidad sobre activos ROA de la empresa pasó de un desempeño del 17,83\% en 2010 al $25,13 \%$ en 2014 mejorando la rentabilidad obtenida por el uso operativo de la organización, lo que evidencia mayor eficiencia en el uso de la infraestructura instalada de la empresa. De igual forma, la organización ha mantenido sus niveles de crecimiento en cuanto a utilidades entre los años 200 a 2014, con un comportamiento favorable con referencia al del sector en los periodos 2010,2012 y 2014 lo cual evidencia su adaptación a las condiciones de medio y sector que incluso supera las expectativas de desarrollo del mismo.

En cuanto a la consolidación de la estructura patrimonial, la empresa ha reducido su posibilidad de mantenimiento del mismo ante la caída de precios, tanto de los productos como de los semovientes en el mercado nacional y en el internacional, que ha llevado a valoraciones de los activos inferiores a los niveles mantenidos entre los años 2010 a 2012, pasando a márgenes negativos en los dos últimos años. Lo descrito corresponde con los resultados en cuanto a margen bruto y neto de ventas, tal como se presenta en las Gráficas 3 y 4.
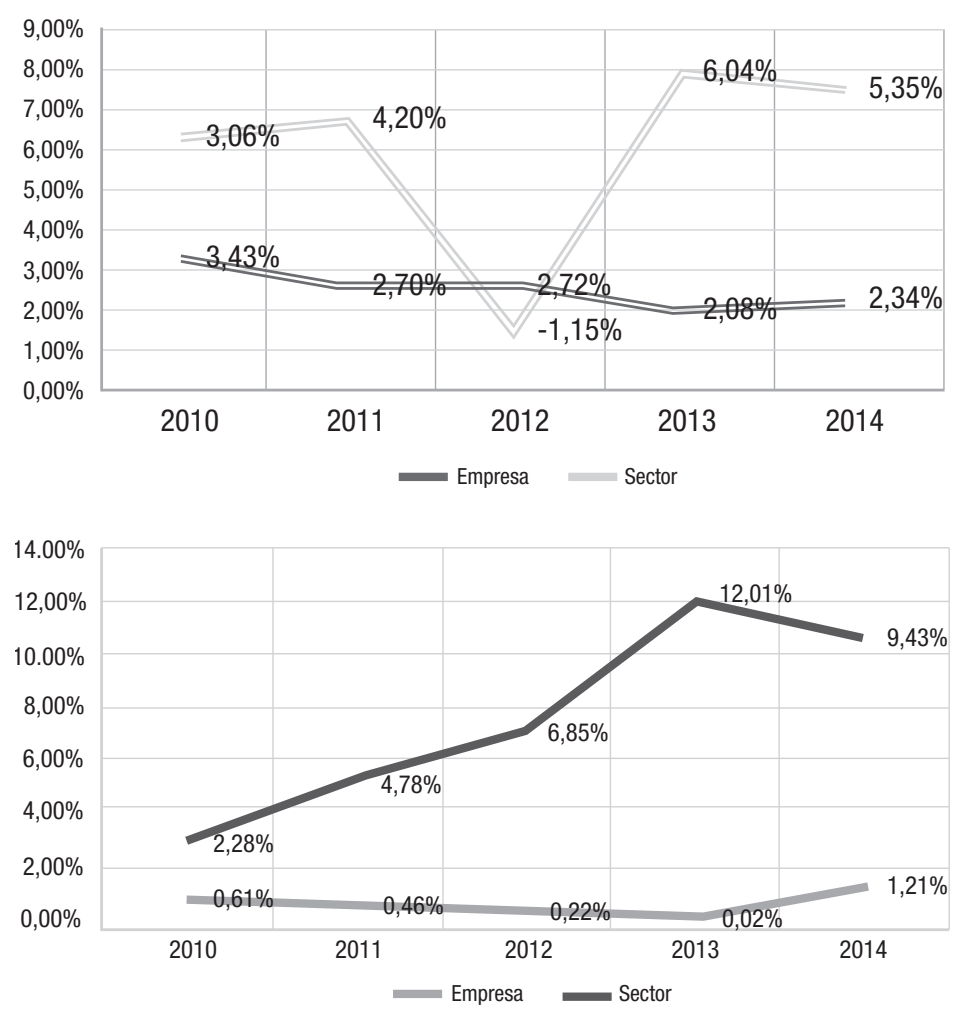

Gráficos 3 y 4. Utilidad operacional y utilidad neta en ventas

Fuente: El autor 
Al mostrar los resultados agregados de los subsectores, el desempeño en utilidades netas y operacionales del sector presenta rendimientos que sobrepasan la capacidad operativa de la empresa, no obstante como empresa líder, la Compañía Cerdos del Valle S.A. mantiene sus niveles de utilidad operacional entre el 3,43\% y el 2,34\% y la utilidad neta sobre ventas anuales entre el 0,61\% y el 1,21\% para el periodo de análisis. Aun con los factores externos del sector, la empresa ha conseguido mantenerse en el mercado generando flujos de efectivo positivos y permitiendo la generación de valor organizacional.

Como medidas de este aspecto, a continuación se presenta el desempeño de la empresa con referencia a los inductores de valor Ebitda y Generación Interna de fondos Gif. (Gráficos 5 y 6).
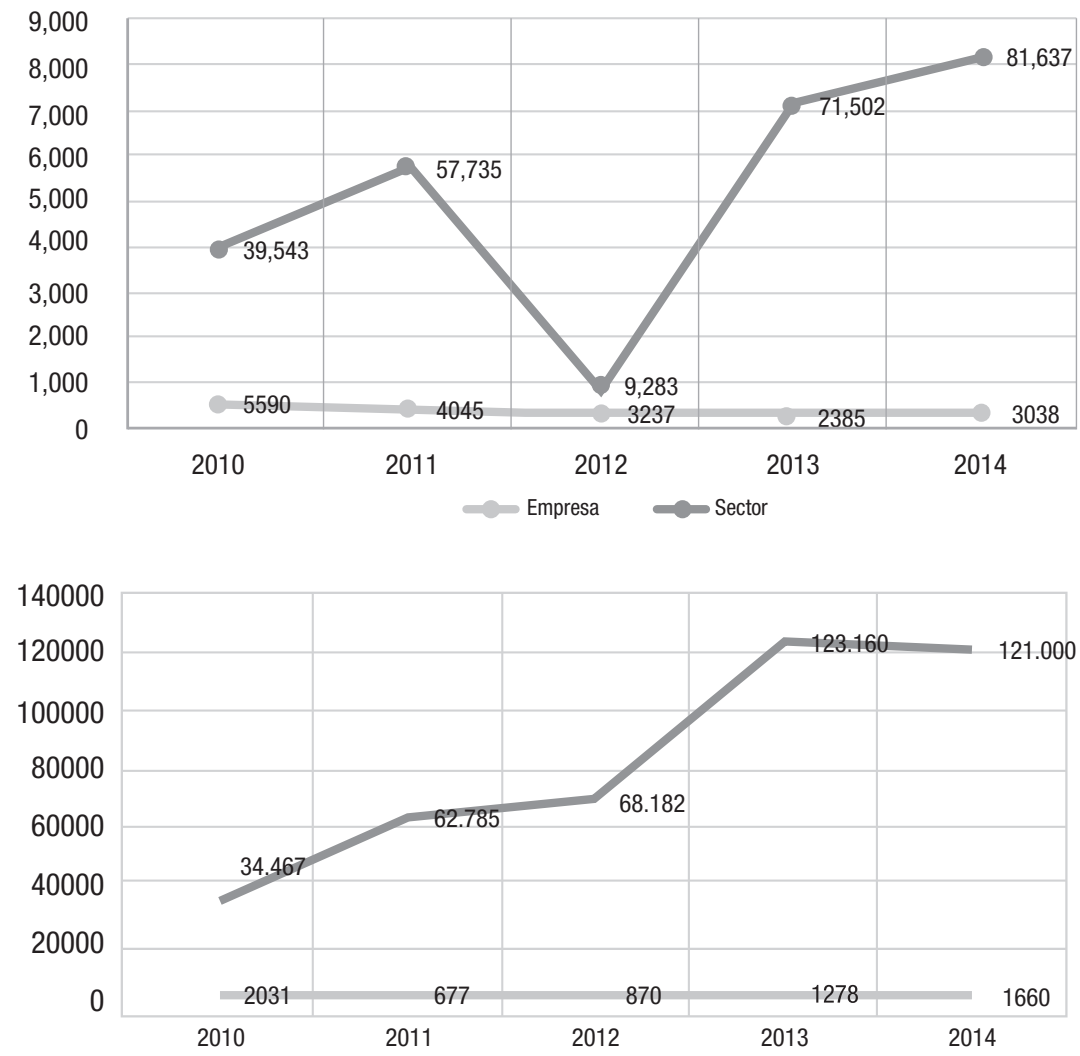

Gráficos 5 y 6. Inductores de valor Ebitda y generación interna de fondos (GIF)

Fuente: El autor

El inductor operativo de valor Ebitda permite indicar la utilidad operativa que se obtendría antes de considerar los gastos que no implican desembolso de efectivo, ni lo implicarán 
en el futuro, respecto del sector puede inferirse que al devolver los gastos que no generan salidas de efectivo, tales como depreciaciones y amortizaciones, se sigue obteniendo para Cerdos del Valle S.A. un resultado positivo en cuanto a su capacidad de generación de flujos de efectivo futuros. Aunque el desempeño empresarial no está en el rango de los alcanzados por el sector, su desempeño en cuanto Ebitda es estable, encontrándose en niveles entre 5.590 y 3.038 millones de pesos para los años de análisis. De otra parte, la generación interna de Fondos Gif en la empresa es consecuente con el indicador previo toda vez que la empresa ha mantenido sus posibilidades de generación de flujos positivos de caja entre los 2.031 y 1.660 millones de pesos para los años de análisis. La generación de flujos de efectivo presentes y futuros permite a la empresa garantizar su continuidad en el tiempo, a la vez que se convierte en un indicador de capacidad de convertir en flujos de efectivo positivos los factores de producción organizacionales.
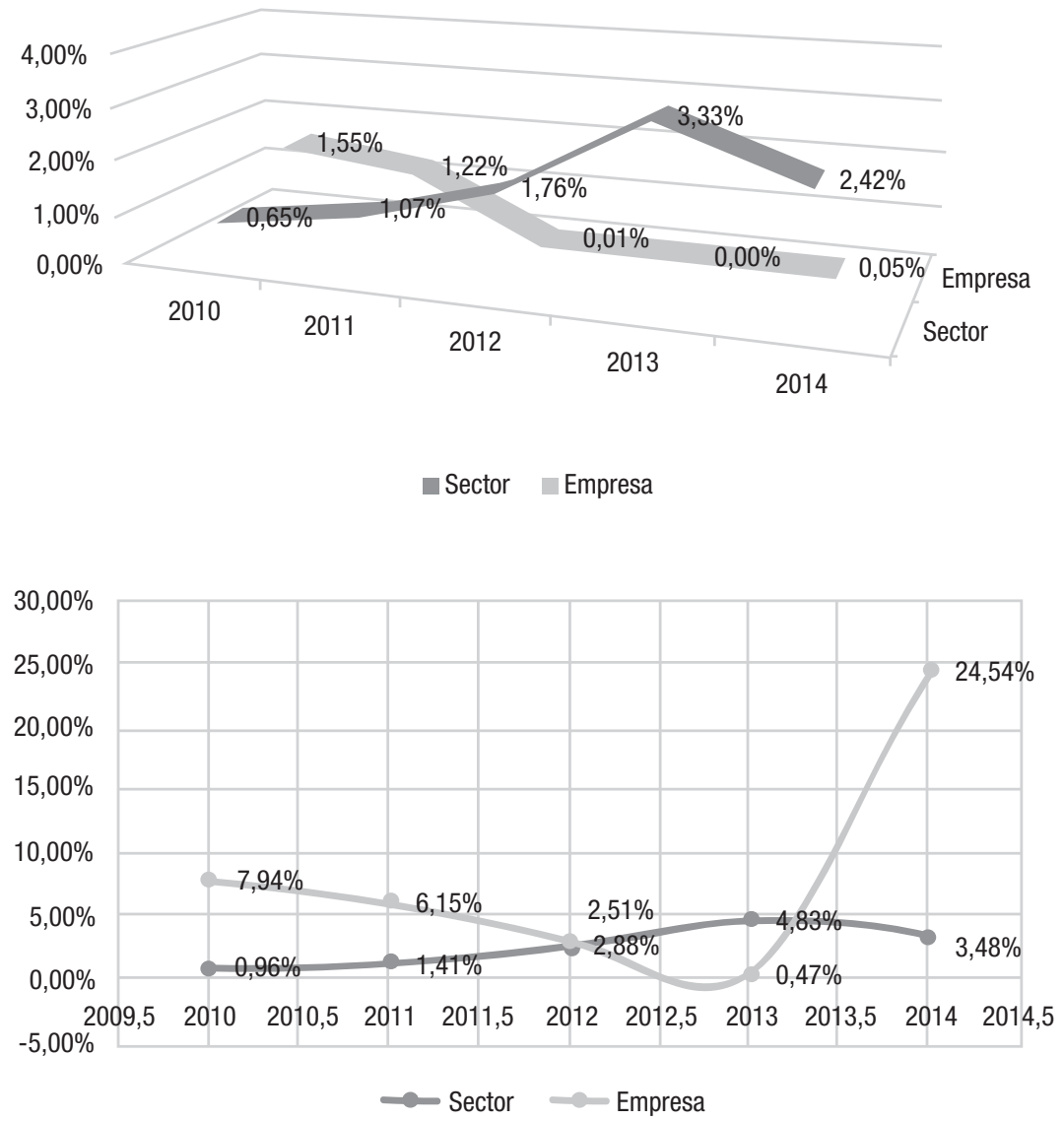

Gráficos 7 y 8. Rentabilidad de activos ROA y rentabilidad neta patrimonio ROE

Fuente: El autor 
En cuanto a rentabilidad los indicadores de rentabilidad operativa de activos ROA, neta de patrimonio ROE y contribución financiera de la empresa respecto del sector se presentan en los gráficos 7 y 8 previamente. Mientras el sector muestra una tendencia al alza en cuanto a rentabilidad, pasando el $0,65 \%$ en el 2010 año 2,42\% en el 2014, la empresa revela una tendencia a la baja del 1,55\% al 0,05\% respectivamente. Lo anterior se explica por la reducción de la capacidad de producción respecto de la capacidad instalada, causada por una contracción de la demanda externa del producto, especialmente en los últimos dos años que implica presencia de activos ociosos en la empresa ante niveles de producción por debajo de los históricamente presentados.

De manera complementaria se muestra una rentabilidad neta del patrimonio ROE, aspecto que llama principalmente la atención dado su desempeño en los últimos año, pasando del 0,47\% en el 2013 al 24,54\% en el 2014 lo que indica la capacidad de la empresa para generar rentabilidad para sus socios, por cuanto los niveles de desempeño alcanzados se encuentran bastante por encima de los del sector, que se ubican en un 3,48\% para el año 2014, convirtiéndola en una empresa que genera rentabilidad por la fuente operativa y financiera, lo que se hace evidente en un incremento en la contribución financiera que pasa del 0,40\% al 9,74\% en los mismos periodos de análisis.

Finalmente, en cuanto al ciclo de efectivo, la empresa se encuentra un $42 \%$ por debajo del ciclo del sector siendo de 40 días con referencia a 197 para el año 2014. Esto implica que Cerdos del Valle S.A. como empresa líder maneja ciclos de caja que le permiten flujos de efectivo continuos desde y hacia la empresa, con condiciones de liquidez propicias para garantizar sus recursos operativos y responder con suficiencia a sus compromisos financieros.

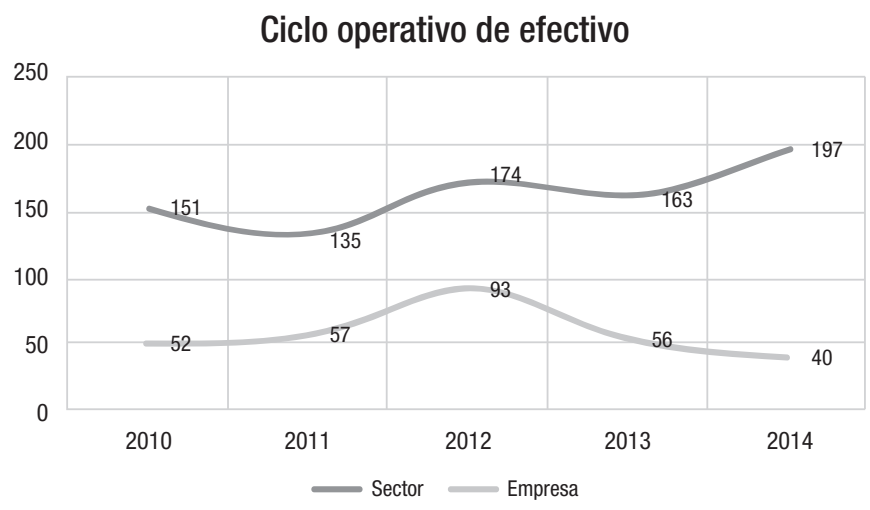

Gráfico 9. Ciclo operativo de efectivo

Fuente: El autor 


\section{Conclusiones}

Con referencia a la asociatividad y su influencia en el desarrollo, lo expresado por los autores refuerza el argumento de la necesidad de incorporación tanto en las políticas públicas como la articulación con los actores de desarrollo para posibilitar el tránsito de lo deseable a lo socialmente realizable.

De manera complementaria la compilación normativa, tanto nacional como departamental permite entrever el propósito gubernamental de fortalecimiento del sector, al incluirlo como apuesta productiva y sector promisorio para el desarrollo en los niveles territoriales considerados.

En cuanto a competitividad la cohesión entre miembros del sector permite mejorar el acceso a los mercados internacionales, toda vez que su fortalecimiento garantiza condiciones para dar cobertura a las demandas de los clientes al tiempo que mejora la participación e integración entre miembros del sector con una mayor participación en la economía nacional.

A partir de lo expresado puede concluirse que la empresa Cerdos del Valle S.A. como líder del sector ganadero propone una estrategia de generación de valor que se percibe tanto en sus decisiones operativas como financieras y de gestión empresarial, que le permiten incorporar las variables externas del sector a la conjugación de la infraestructura organizacional, incorporando variables como la innovación y diversificación para hacer frente a los retos y desafíos del sector ganadero, convirtiéndolos en fortalezas y oportunidades en su gestión organizacional para el cumplimiento de los objetivos financieros pretendidos tanto interna como externamente.

El liderazgo del sector, por tanto, se explica por la convergencia organizacional de aspectos administrativos, operativos y financieros que conjugados con estrategias empresariales le permiten a Cerdos del Valle S.A. mantenerse con indicadores que sobrepasan las expectativas del sector, hábida cuenta de las decisiones en materia de inversión y financiación acordes con las propuestas de generación de valor, para los grupos de interés tanto internos como externos de la empresa.

\section{Conflicto de intereses}

La autora declara no tener ningún conflicto de intereses.

\section{Referencias bibliográficas}

1. Atria, R. (2003). Cepal, Seminario Taller "Capital Social, Herramienta para los Programas de Superación de la Pobreza Urbana y Rural”. La Dinámica del Desarrollo del Capital Social: Factores Principales. Sala de Conferencias II Cepal, S/P.

2. Departamento del Quindío. (2012 ). Ordenanza Número 018 del 30 de mayo de 2012, por medio de la cual se aprueba el Plan de Desarrollo Departamental 2012-2015. Armenia: Gaceta Departamental. 
3. Emis. (2015). Informe Sectorial Ganadero. Londres: Euromoney Institutional Investor Company.

4. García, O. (2000) Administración Financiera, Fundamentos y Aplicaciones.

5. Kliksberg, B. (1999). Capital Social y Cultura - Claves Olvidadas del Desarrollo. Revista de la Cepal Vol. 69, 85-102

6. Londoño Franco, I. C., \& Sánchez Vásquez, L. M. (2016). Identificación de los encadenamientos más promisorios del sector agroindustrial del departamento del Quindío y el grado de asociatividad entre sus actores en el periodo 2008-2011. Armenia: Editorial Eam.

7. Ríos, A. y Ríos, J. (1999). Capital Social y Democracia: Una revisión crítica.

8. Rodríguez Figueroa, H. (2016) Material seminario de economía y finanzas Dba Iv Cohorte, AIU.

9. Putnam. Colección Política y Gobierno, Vol. 6, 513-528

10. Sekn. (2009, 24 de diciembre). Gestión efectiva de emprendimientos sociales. Lecciones extraídas de empresas y organizaciones de la Sociedad Civil en Iberoamérica. Recuperado de http://Idbdocs.Iadb.Org/Wsdocs/Getdocument Aspx?Docnum=1579031

11. Sencilio, G. (2008, 22 de octubre). En Módulo I del III Curso Internacional Virtual de responsabilidad social en el agro. Recuperado de http://www.Eticagro.Org/Campus/ Index.Php. 